\title{
ON THE $L$-HOMOMORPHISMS OF FINITE GROUPS
}

BY

\section{MICHIO SUZUKI}

Let $G$ be a finite group. We shall denote by $L(G)$ the lattice formed by all subgroups of $G$. A homomorphic mapping from $L(G)$ onto a lattice $L$ is called an $L$-homomorphism from $G$ onto $L$.

In his previous paper (Suzuki [5](1)), dealing with $L$-isomorphisms of finite groups, the author determined the structure of groups, $L$-isomorphic to a $p$-group, and proved that groups $L$-isomorphic to a solvable or a perfect group are also solvable or perfect respectively. In this paper we shall generalize these results to the case of $L$-homomorphisms and study the relations between $L$-homomorphisms and $L$-isomorphisms. In particular, we shall determine all $L$-homomorphisms from a perfect group, and as an application, we shall also determine the neutral elements of $L(G)$.

$L$-homomorphisms of finite groups were first considered by $\mathrm{P}$. Whitman [6], who dealt with the case when $L$ is the subgroup lattice of a cyclic group. His result will be sharpened to Theorem 1 in $\$ 1$ which will play a fundamental rôle in our study.

\section{SOME REMARKS ON $L$-HOMOMORPHISMS}

Let $G$ be a group and $\phi$ be an $L$-homomorphism from $G$ onto a lattice $L$. A set of elements of $L(G)$, which is mapped onto a fixed element of $L$, forms a convex sublattice $\left({ }^{2}\right)$ of $L(G)$, and in particular elements mapped to the least (greatest) element $0(I)\left(^{3}\right)$ of $L$, form a (dual) ideal of $L(G)$. The greatest (least) element of such a (dual) ideal is called the "lower (upper) kernel," or shortly " $l$ - $(u-)$ kernel" of $\phi$ in $G$.

First we shall prove the following lemma.

Lemma 1. [Cf. 6]. Let $G$ be a group and $\phi$ be an L-homomorphism from $G$ onto a chain $C_{n}$ of dimension $n$. Then there are two subgroups $N$ and $G_{0}$ of $G$ and a prime number $p$ with the following properties:

(1) $N$ is a Sylow p-complement $\left.{ }^{4}\right)$ of $G$,

(2) a p-Sylow subgroup $S_{p}$ contains $G_{0}$ and is cyclic or a generalized quaternion group (g. q. group),

Received by the editors April 8, 1950.

(1) Numbers in brackets refer to the bibliography at the end of the paper.

$\left({ }^{2}\right)$ For general lattice theory, see Birkhoff [2].

(3) In the following we always denote by $0(I)$ the least (greatest) elements of various lattices and do not mention it particularly, if there is no risk of misunderstanding.

(4) Sylow $p$-complements of a group of order $p^{n} g,(p, g)=1$, are subgroups of index $p^{n}$. Cf. Suzuki $[5$, footnote 8$]$. 
(3) If the order of $G_{0}$ is $p^{m}$, we have $m \geqq n$, and

(4) If $S_{p}$ is a g. q. group, the order of $G_{0}$ is 2 .

Conversely if there are normal subgroups $N$ and $G_{0}$ of $G$ and a prime number $p$ with the properties (1)-(4), then $L(G)$ is homomorphic to a chain $C_{n}$ of dimension $n$.

Proof. Denote by $G_{0}$ the $u$-kernel of $\phi . G_{0}$ has only one maximal subgroup and hence $G_{0}$ is a cyclic group of prime power order. Let $p^{m}$ be this order. Take a Sylow subgroup $S_{p}$ of $G$ containing $G_{0}$. If there were a noncyclic subgroup $V$ of $S_{p}$ covering $G_{0}, V$ would be $L$-homomorphic to $C_{n}$. Since the factor group $V / \Phi(V)\left(^{5}\right)$ is a $P$-group, there would exist at least two maximal subgroups $M_{1}$ and $M_{2}$ of $V$, different from $G_{0}$. Both $\phi\left(M_{1}\right)$ and $\phi\left(M_{2}\right)$ would be maximal elements of $C_{n}$, and we should, therefore, have $\phi(V)=\phi\left(M_{1} \cup M_{2}\right)$ $=\phi\left(M_{1}\right) \cup \phi\left(M_{2}\right) \neq I$, which is clearly a contradiction. Hence all subgroups of $S_{p}$ covering $G_{0}$ are cyclic and $S_{p}$ has only one subgroup of order $p . S_{p}$ must be cyclic or a g. q. group [cf. 7, p. 112].

Take a $q$-Sylow subgroup $S_{q}$, where $q$ is any prime factor of the order of $G$ other than $p$. We have $\phi\left(S_{q}\right) \cap \phi\left(S_{p}\right)=0$ because $S_{q} \cap S_{p}=e$. This implies that $\phi\left(S_{q}\right)=0$. Put $N=\bigcup_{q \neq p} S_{q}$, where $q$ runs through all prime factors of the order of $G$ except $p$. Then $N$ is clearly self-conjugate. Take a normalizer $N_{q}$ of $S_{q}$ in $G$, then we have $N_{q} \cdot N=G$. Hence $N_{q}$ contains a $p$-Sylow subgroup of $G$. Choosing a suitable $q$-Sylow subgroup $S_{q}$ we may assume that $N_{q} \supseteq S_{p} \supseteq G_{0}$. We shall prove that $G_{0}$ is self-conjugate in $H=G_{0} \cdot S_{q}$, using induction on the dimension of the interval $H / G_{0}$. We take a maximal subgroup $M$ of $H$ containing $G_{0}$, then $M \cap S_{q}$ is self-conjugate in $H . H / M \cap S_{q}$ is $L$-homomorphic to $C_{n}$ because $\phi\left(M \cap S_{q}\right)=0$ and $\phi(H)=I$. Hence we have only to prove our assertion in the case where $G_{0}$ is maximal. If $G_{0}$ were not self-conjugate in such a case, there would be at least two subgroups $G_{1}$ and $G_{2}$ of $H$, conjugate to and different from $G_{0}$. We should then have $\phi\left(G_{1}\right)$ $=\phi\left(G_{2}\right) \neq I$, which gives the contradiction that $\phi(H)=\phi\left(G_{1}\right) \cup \phi\left(G_{2}\right) \neq I$. Hence $G_{0}$ is self-conjugate in $H$. Since $q$ is an arbitrary prime factor other than $p$, this implies that $G_{0}$ is self-conjugate in $G$ and that $G_{0}$ is elementwise permutable with $N$. By the definition of $N$ this implies that $N \cap G_{0}=e$ and $N \cdot S_{p}=G$. The former part of our lemma now follows immediately.

Conversely, suppose $G$ to have such a structure. Then $G$ is proved to be $L$-homomorphic to a chain as follows.

When $S_{p}$ is a g. q. group, the mapping $\phi$ from $L(G)$ onto the two-element lattice $C_{2}$ defined by

$$
\phi(V)= \begin{cases}I & \text { if the order of } V \text { is even, } \\ 0 & \text { if the order of } V \text { is odd, }\end{cases}
$$

(5) We mean by $\Phi(V)$ the $\Phi$-subgroup of $V$, which is defined to be the intersection of all maximal subgroups of $V$. Cf. Zassenhaus [7, p. 44]. 
is an $L$-homomorphism from $G$ onto $C_{2}$. For subgroups of even order contain $G_{0}$ and those of odd order are contained in $N$.

When $S_{p}$ is cyclic, the mapping $\phi$ from $L(G)$ onto the chain $C_{m}$ of dimension $m$ defined by

$$
\phi(V)=a, \quad . \quad\left(\nu=\min (m, \lambda), p^{\lambda} \|(V: e)\right)
$$

is an $L$-homomorphism from $G$ onto $C_{m}$, where $a_{\nu}$ is the element of $C_{m}$ with dimension $\nu$, and $\lambda$ is the exact power of $p$ dividing the order of $V$. For $G_{0} \cup N$ is $L$-decomposable, and subgroups of order $p^{\mu} g$ with $\mu \geqq m((p, g)=1)$ contain $G_{0}$. Hence $G$ is clearly $L$-homomorphic to a chain $C_{n}$ with $n \leqq m$. Note that the mapping $\phi$ defined above is equivalent to the mapping $U \rightarrow G_{0}$ $\cap U$ from $L(G)$ onto a chain $L\left(G_{0}\right)$.

By this lemma we can easily generalize Whitman's theorem as follows.

Theorem 1. A group $G$ is L-homomorphic to a cyclic group $G^{\prime}$ of order $\prod_{i=1}^{n} q_{i}^{e_{i}}$ if and only if there exist prime numbers $p_{i}(i=1,2, \cdots, n)$ and two normal subgroups $G_{0}$ and $N$ with the following properties:

(1) $p_{i} \neq p_{j}(i \neq j)$,

(2) the order of $G$ is $\prod_{i=1}^{n} p_{i}^{f_{i}} \cdot g,\left(p_{i}, g\right)=1(i=1,2, \cdots, n)$,

(3) the order of $G_{0}$ is $\prod_{i=1}^{n} p_{i}^{a_{i}}$ with $f_{i} \geqq a_{i}(i=1,2, \cdots, n)$,

(4) $N$ is of order $g$ and the factor group $G / N$ is a nilpotent group whose p-Sylow subgroups are cyclic, or a g. q. group, and

(5) if $p_{i}=2$ and if a 2-Sylow subgroup is a g. q. group, then $a_{i}=e_{i}=1$.

Proof. The subgroup lattice $L\left(G^{\prime}\right)$ of a cyclic group $G^{\prime}$ is a direct product of chains, so that there are natural homomorphisms $\psi_{i}(i=1,2, \cdots, n)$ from $L\left(G^{\prime}\right)$ onto its direct components. Let $\phi$ be the homomorphism from $L(G)$ onto $L\left(G^{\prime}\right)$. Then $\psi_{i} \phi$ is clearly a homomorphism from $L(G)$ onto a chain. Hence $G$ has a prime factor $p_{i}$ and two normal subgroups $G_{i}$ and $N_{i}$ with the properties given in Lemma 1 . Now we have clearly $p_{i} \neq p_{j}(i \neq j)$. Put $G_{0}$ $=\bigcup G_{i}$ and $N=\cap N_{i}$, then $G_{0}$ and $N$ satisfy the properties of Theorem 1 .

Conversely, suppose that $G$ has prime factors $p_{i}(i=1,2, \cdots, n)$ and two normal subgroups with the above properties. Then $G$ has the Sylow $p_{i}$-complement $N_{i}$ and $G_{0}$ is nilpotent. Let $G_{i}$ be a $p_{i}$-Sylow subgroup of $G_{0}$. Then both $N_{i}$ and $G_{i}$ are self-conjugate in $G$. By Lemma $1, G$ is $L$-homomorphic to $L\left(G_{i}\right)$. We shall denote by $\phi_{i}$ this $L$-homomorphism from $G$ onto $L\left(G_{i}\right)$. We have then

$$
\phi_{i}\left(G_{j}\right)=0
$$

Let $\phi_{0}$ be a mapping from $L(G)$ into a direct product $L=L\left(G_{1}\right) \times \cdots \times L\left(G_{n}\right)$ defined by

$$
\phi_{0}(V)=\left(\phi_{1}(V), \cdots, \phi_{n}(V)\right) .
$$

$\phi_{0}$ is clearly an $L$-homomorphism from $G$ into $L$, and in virtue of $\left(^{*}\right)$ it is surely onto $L$. As is easily proved, there exists a homomorphism $\psi$ from $L$ 
onto $L\left(G^{\prime}\right)$ of a cyclic group $G^{\prime}$ of order $\prod q_{i}^{e_{i}} \cdot \psi \phi_{0}$ is clearly an $L$-homomorphism from $G$ onto $L\left(G^{\prime}\right)$. q.e.d.

REMARK. The $l$-kernel and the $u$-kernel of $\phi$ are both self-conjugate, if $L$ is a chain.

We obtain now the following two theorems.

THEOREM 2. Let $G$ be a group, and $\phi$ be an L-homomorphism from $G$ onto a lattice $L$. Then the l-kernel of $\phi$ is self-conjugate in $G$.

Proof. The greatest element of $L$ is represented as a join of elements $l_{i}$ such that the intervals $l_{i} / 0$ are chains. Let $l_{1}, \cdots, l_{n}$ be all such elements of $L$. Take a subgroup $V_{i}$ of $G$ such that $\phi\left(V_{i}\right)=l_{i}(i=1,2, \cdots, n)$ and let $V_{i}$ be maximal under this condition. Then we have $\cup_{i=1}^{n} V_{i}=G$. Let $E$ be the $l$-kernel of $\phi$. Then we have $\phi\left(V_{i} \cup E\right)=\phi\left(V_{i}\right) \cup \phi(E)=\phi\left(V_{i}\right)=l_{i}$, which implies that $V_{i} \cup E=V_{i}$ or $V_{i} \supseteq E$. Hence $E$ is self-conjugate in $V_{i}$, as the $l$-kernel of $\phi\left({ }^{6}\right)$ between $V_{i}$ and $l_{i} / 0 . E$ is, therefore, self-conjugate in $G$.

THEOREM 3. Under the same assumptions as in Theorem 2, the u-kernel $G_{0}$ of $\phi$ is also self-conjugate in $G$.

Proof. We shall prove our theorem by induction on the dimension of $L$. Since the greatest element of the interval $G / G_{0}$ is represented as a join of join-irreducible (that is, covering only one element) elements, we may assume that $G$ has only one maximal subgroup containing $G_{0}$. If no other maximal subgroup exists, $G$ is cyclic and our theorem is obvious. If there exists another maximal subgroup $M, \phi(M)$ must be a dual atom of $L$. By the hypothesis of induction, the $u$-kernel $M_{0}$ of $\phi$ in $M$ is self-conjugate in $M$. Since $\phi\left(M \cap G_{0}\right)=\phi\left(G_{0}\right) \cap \phi(M)=\phi(M)$, we have $M \cap G_{0} \supseteq M_{0}$. Take any element $a$ of $M$, then $a \cdot G_{0} \cdot a^{-1} \cup M=G$. Hence we have $\phi\left(a \cdot G_{0} \cdot a^{-1}\right) \cup \phi(M)$ $=I$. On the other hand, we have $\phi\left(a \cdot G_{0} \cdot a^{-1}\right) \supseteq \phi\left(a \cdot M_{0} \cdot a^{-1}\right)=\phi\left(M_{0}\right)=\phi(M)$. Hence we have $I=\phi\left(a \cdot G_{0} \cdot a^{-1}\right)$ which implies that $a \cdot G_{0} \cdot a^{-1} \supseteq G_{0}$ and hence $a \cdot G_{0} \cdot a^{-1}=G_{0} . G_{0}$ is therefore self-conjugate in $G$. q.e.d.

\section{GROUPS WHICH ADMIT PROPER $L$-HOMOMORPHISMS}

An $L$-homomorphism is called proper if it is neither an $L$-isomorphism nor a trivial $L$-homomorphism. Otherwise we call it improper. We shall say that a group $G$ admits a proper $L$-homomorphism when there exists a lattice $L$ and an $L$-homomorphism from $G$ onto $L$ which is proper. In this section we shall consider the structure of groups which admit proper $L$-homomorphisms. First we shall prove the following lemma.

Lemma 2. If a p-group $G$ admits a proper L-homomorphism, $G$ is either a cyclic group or a g. q. group.

(6) Strictly speaking, it is a contraction of $\phi$ on to $U$. We shall, in this paper, not distinguish a contraction of $\phi$ from $\phi$, as long as no confusion arises. 
Proof. Let $\phi$ be a proper $L$-homomorphism from $G$ onto a lattice $L$. If the $u$-kernel $G_{0}$ of $\phi$ differs from $G$, we can prove our lemma in a similar way as in the proof of Lemma 1. In the following we shall assume that $G_{0}=G$, and prove our lemma by induction on the order of $G$. Since $G$ is a $p$-group, $L$ satisfies the Jordan-Dedekind chain condition. Since $\phi$ is a proper $L$-homomorphism, the dimension of $L$ is different from that of $L(G)$. Hence every maximal subgroup of $G$ admits a proper $L$-homomorphism, that is, that induced by $\phi$. By the hypothesis of induction, every maximal subgroup of $G$ contains only one subgroup of order $p$. Hence $G$ is either a $P$-group of order $p^{2}$, or one of the types stated in Lemma 2 . On the other hand, $P$-groups admit no proper $L$-homomorphism. Hence we have our lemma.

Let $\phi$ be again a proper $L$-homomorphism from $G$ onto $L$. We shall denote by $E$ the $l$-kernel and by $G_{0}$ the $u$-kernel of $\phi$ and put $E_{0}=G_{0} \cap E$ and $G_{1}=G_{0} \cup E$. Then these four subgroups $E, G_{0}, E_{0}$, and $G_{1}$ are all self-conjugate. Hence we may consider the factor group $\bar{G}_{1}=G_{1} / E_{0}$ which is clearly a direct product of $\bar{G}_{0}=G_{0} / E_{0}$ and $\bar{E}=E / E_{0}$. These notations will be fixed throughout this section.

We shall prove the following propositions.

(a) The groups $\bar{G}_{0}$ and $\bar{E}$ have mutually prime orders.

Proof. If the orders of $\bar{G}_{0}$ and $\bar{E}$ had a common prime factor $p$, there would exist two subgroups $V_{1}$ and $V_{2}$ of $\bar{G}_{0}$ and $\bar{E}$ respectively whose orders are $p$. Hence $V_{1} \cup V_{2}$ would contain another subgroup $V$ such that $\bar{G}_{0} \cap V=e$ and $\bar{E} \cap V=e$. The first equality implies that $\phi(V)=0$ and $V \subseteq \bar{E}$, but the second equality implies that $\bar{E} \nsupseteq V$. This is a contradiction. q.e.d.

(b) $\Phi\left(G_{0}\right)$ contains $E_{0}$.

Proof. Take any maximal subgroup $M$ of $G_{0} . \phi(M)$ must be a dual atom of $L$. We have $\phi\left(M \cup E_{0}\right)=\phi(M) \cup \phi\left(E_{0}\right)=\phi(M) \cup 0=\phi(M)$ and hence $M$ $\cup E_{0}=M$. This implies that $M \supseteq E_{0}$ and that $\phi\left(G_{0}\right) \supseteq E_{0}$. q.e.d.

(b') (Cf. [5, Lemma 4].) $E_{0}$ is nilpotent, and if a prime number $p$ divides the order of $E_{0}, p$ divides also that of $\bar{G}_{0}$.

(c) $G_{1}$ is a direct product of $G_{0}$ and another group $N . N$ is isomorphic to $\bar{E}$ and its order is relatively prime to that of $G_{0}$.

Proof. By $\left(\mathrm{b}^{\prime}\right)$ and (a) the order of $E_{0}$ is relatively prime to that of $E / E_{0}$. Hence by a theorem of Schur (cf. [7, p. 125]) there exists a subgroup $N$ of $E$ such that $N \cup E_{0}=E$ and $N \cap E_{0}=e$. Take the normalizer $N^{*}$ of $N$ in $G$. Then we have $N^{*} \cup E=G$, since $E_{0}$ is nilpotent by (b') (cf. [7, p. 125]). Hence we have $I=\phi(G)=\phi\left(N^{*} \cup E\right)=\phi\left(N^{*}\right) \cup \phi(E)=\phi\left(N^{*}\right)$. This implies that $N^{*} \supseteq G_{0}$. Hence $N^{*} \supseteq G_{0} \cup N=G_{0} \cup E_{0} \cup N=G_{0} \cup E=G_{1}$. It follows then that $N$ is a normal subgroup of $G . G_{1}$ is clearly a direct product of $G_{0}$ and $N$, and $N$ is isomorphic to $\bar{E}$.

(d) If a prime number $p$ divides the order of $G / G_{1}$, then $p$ divides that of $G_{1} / E$. Hence the groups $G / N$ and $N$ have mutually prime orders.

Proof. Take any prime factor $p$ of the order of $G / G_{1}$. If $p$ did not divide 
the order of $G_{1} / E$, a $p$-Sylow subgroup $\bar{S}$ of $G / E$ would satisfy the condition $\bar{S} \cap G_{1} / E=e$. We mean by $S$ a subgroup of $G$ corresponding to $\bar{S}$ by the natural homomorphism from $G$ onto $G / E$. Then we should have $S \cap G_{1}=E$ and $\phi(S)=\phi\left(S \cap G_{1}\right)=\phi(E)=0$. This implies that $S \subseteq E$, which gives a contradiction. Hence $p$ divides the order of $G_{1} / E$. q.e.d.

Hence again by Schur's theorem, $G$ contains a subgroup $H$ such that $G$ $=H \cdot N, H \cap N=e$ and $H \supseteq G_{0}$. Now we have, in a similar way as for (b),

(e) $\Phi(H)$ contains $E_{0}$.

Next we shall prove the following proposition.

(f) If $\phi$ induces an improper $L$-homomorphism of every Sylow subgroup of $G$ into $L$, then $H$ is mapped isomorphically onto $L$ by $\phi$ and we have $G=G_{0} \times E$.

Proof. By the assumption of this proposition and by propositions $\left(b^{\prime}\right)$ and (d), we have $E_{0}=e$ and $H=G_{0}$. Our proposition follows then immediately.

By means of proposition (f) we shall deal with a Sylow subgroup in which $\phi$ induces a proper $L$-homomorphism. We shall prove the following propositions.

(g) If a g. q. group $Q$ is mapped by $\phi$ onto a chain of dimension two, $H$ is a direct product of its 2-Sylow subgroup $S_{2}$ and its Sylow 2-complement $K$ In this case, $L$ is also a direct product of $\phi\left(S_{2}\right)$ and $\phi(K)$.

Proof. First, using induction on the order of $G$, we prove that $G$ has a self-conjugate Sylow 2-complement. By Lemma 2, 2-Sylow subgroups of $G$ are g.q. groups. Take any proper subgroup $V$ of $G$. If its 2-Sylow subgroup is cyclic. $V$ has a self-conjugate Sylow 2-complement by a theorem of Burnside (cf. [7, p. 131]). The same holds from the hypothesis of induction if its 2-Sylow subgroup is a g.q. group. Hence every proper subgroup of $G$ has a self-conjugate Sylow 2-complement. By a theorem of Ito $\left.{ }^{7}\right), G$ has also a self-conjugate Sylow 2-complement, or all proper subgroups of $G$ are nilpotent. In the latter case, if its Sylow 2-complement were not self-conjugate, $G$ would be of order $p^{\alpha} 2^{\beta}$ ( $p$ is a prime greater than 2 ). The structure of such a group has been completely determined by Iwasawa $\left({ }^{8}\right)$. We can prove by direct examinations that our assumption does not hold in this case. Hence $G$ has a self-conjugate Sylow 2-complement.

Next using again induction on the order of $H$, we prove that $H$ is a direct product of its 2-Sylow subgroup and the Sylow 2-complement. We shall denote by $K$ the Sylow 2-complement of $H$ and assume for a while that the $l$-kernel of $\phi$ coincides with $e$. Considering normalizers of Sylow subgroups

$\left({ }^{7}\right)$ Cf. N. Ito, Zenkoku Sizyô Sûgaku-Danwa-Kai 2-93 (1948) (In Japanese). His theorem asserts that if all proper subgroups of a finite group $G$ have the self-conjugate Sylow $p$-complement, then $G$ has also a self-conjugate Sylow $p$-complement except when all proper subgroups are nilpotent. His proof is a slight modification of the proof given in K. Iwasawa, Proc. of P-M. Soc. of Japan, 3-23 (1941).

(8) Cf. A paper of Iwasawa quoted in footnote 7 . 
of $K$, we can assume $K$ to be a $p$-group $(p>2)$. If $K$ is cyclic, the centralizer of $K$ contains the center $Z$ of a 2 -Sylow subgroup $S_{2}$. Since $\phi(K \cup Z)=\phi(K)$ $\cup \phi(Z)=\phi(K) \cup \phi\left(S_{2}\right)=\phi(H), K Z$ contains the $u$-kernel of $\phi$ and it is a direct product of $K$ and $Z$. Hence we have $L=(\phi(K) / 0) \times(\phi(Z) / 0)$. Let $\psi$ be the natural homomorphism from $L$ onto $\phi(K) / 0$. Then $\psi \phi$ is an $L$-homomorphism from $H$ onto $\phi(K) / 0$ and $S_{2}$ is the $l$-kernel of $\psi \phi$, since we assumed the $l$ kernel of $\phi$ to coincide with $e$. Hence by Theorem 2, $S_{2}$ is self-conjugate in $H$ and we have $H=K \times S_{2}$.

If $K$ is not cyclic, $\phi$ induces an $L$-isomorphism from $K$ into $L$ by Lemma 2 . We can, therefore, assume also that $S_{2}$ is maximal. If the center $Z$ of $S_{2}$ is self-conjugate in $H, S_{2}$ is self-conjugate in the same way as above. If $Z$ were not self-conjugate in $H, Z$ would be conjugate to another group $Z_{1}$. $Z_{1}$ would be the center of a 2-Sylow subgroup $Q$ and $Q \neq S_{2}$. Then we should have $\phi\left(Z \cup Z_{1}\right)=\phi(Z) \cup \phi\left(Z_{1}\right)=\phi\left(S_{2}\right) \cup \phi(Q)=\phi(H)$ and hence $Z \cup Z_{1} \supseteq K$. This implies that $K$ would be cyclic, which gives a contradiction.

If the $l$-kernel $E_{0}$ of $\phi$ in $H$ differs from $e$, the $l$-kernel of $\phi$ in $H / E_{0}$ coincides with $e$. Hence the 2-Sylow subgroup $\bar{V}$ of $H / E_{0}$ is self-conjugate. Let $V$ be a subgroup of $H$ corresponding to $\bar{V}$ by the natural homomorphism from $H$ onto $H / E_{0}$. Then $V$ is self-conjugate in $H$. Take the normalizer $N_{2}$ of a 2-Sylow subgroup $S_{2}$ of $H$. Then we have $N_{2} V=H$ because $S_{2} \subseteq V$. On the other hand, we have $N_{2} V=N_{2} \cup S_{2} \cup E_{0}=N_{2} \cup E_{0}$. Hence we have $N_{2} E_{0}=H$, which implies that $H=N_{2}$ by $(\mathrm{e})\left({ }^{9}\right)$. Hence $S_{2}$ is self-conjugate and we have $H=S_{2} \times K$. q.e.d.

(h) If $\phi$ induces a proper $L$-homomorphism from a cyclic $p$-Sylow subgroup $S$ into $L$, then $G$ has a self-conjugate Sylow $p$-complement.

Proof. We shall prove that $S$ is contained in the center of its normalizer. If this is done, our proposition follows from a theorem of Burnside (cf. [7, p. 131]). Choosing a suitable subgroup of $G$, we may assume $S$ to be self-conjugate. We shall then prove that $G$ is a direct product of $S$ and the Sylow $p$ complement $K$. Using induction on the order of $G$ we have only to prove our assertion assuming $K$ to be a cyclic group of prime power order, that is, $K=\{b\}$. Put $S=\{a\}$, then we have $b \cdot a \cdot b^{-1}=a^{r}$. If $r \neq 1(\bmod$ the order of $a)$, $G$ would admit no proper $L$-homomorphism, against our assumption. Hence we have $r \equiv 1$ and $G=K \times S$. q.e.d.

By propositions $(\mathrm{g})$ and $(\mathrm{h})$, we get the following propositions.

(i) The factor group $H / G_{0}$ is a nilpotent group each of whose Sylow subgroups is either cyclic or a dihedral group.

(j) If $\phi$ induces a proper $L$-homomorphism of $G_{0} / E_{0}, G_{0}$ contains a normal subgroup $G_{2}$ of $G$ such that the factor group $G_{0} / G_{2}$ is cyclic and $\phi$ induces an $L$-isomorphism of $G_{2} / E_{0}$. Moreover the order of $G_{0} / G_{2}$ is relatively prime to that of $G_{2} / E_{0}$.

( $\left.{ }^{9}\right)$ Let $\Phi$ be the $\Phi$-subgroup of $G$, then $\Phi H=G$ implies $H=G$ for any subgroup $H$ of $G$. Cf. Zassenhaus [7, p. 45]. 
REMARK. If the center $Z$ of a g.q. group $Q$ is mapped onto 0 by $\phi$, and if $\phi(Q) \neq 0, Z$ is clearly self-conjugate by $\left(\mathrm{b}^{\prime}\right)$, since $Z \subseteq E_{0}$. Hence $Z$ is contained in the center of $G$. Conversely if a 2-Sylow subgroup $Q$ of $G$ is a g.q. group and if the center $Z$ of $Q$ is self-conjugate in $G$, then the natural homomorphism from $G$ onto $G / Z$ induces an $L$-homomorphism from $G$ onto $G / Z$ (see Lemma 4 below).

From $\left(b^{\prime}\right),(h)$ and the remark given above we obtain:

(k) $E_{0}$ is a cyclic group contained in the center of $G$.

Proof. By $\left(\mathrm{b}^{\prime}\right)$ and Lemma 2, $E_{0}$ is cyclic. Let $T$ be a $p$-Sylow subgroup of $E_{0}$, and $S$ be a $p$-Sylow subgroup of $G$. $S$ is then cyclic or a g.q. group. If it is a g.q. group, $T$ is contained in the center of $G$ as remarked above. If $S$ is cyclic, $\phi$ induces a proper $L$-homomorphism of $S$. Hence by (h), $G$ has a selfconjugate Sylow $p$-complement $K$. As $T$ is self-conjugate by $\left(\mathrm{b}^{\prime}\right), K \cup T$ is a direct product of $K$ and $T$, which implies that $T$ is contained in the center of $G$. This proves proposition (k).

These propositions may be summarized as follows.

THEOREM 4. If $G$ admits a proper L-homomorphism $\phi$, then $G$ contains a normal subgroup $N$ and a subgroup $H$ such that

(1) $N H=G$ and $N \cap H=e$,

(2) The orders of $N$ and $H$ are relatively prime,

(3) $H$ contains the $u$-kernel $G_{0}$ of $\phi$, and

(4) $N$ is contained in the l-kernel $E$ of $\phi$. Moreover putting $E_{0}=E \cap G_{0}$ we have

(5) $E_{0}$ is a cyclic group, contained in the center of $G$.

The factor group $H / G_{0}$ is a nilpotent group, each of whose Sylow subgroups is either cyclic or a dihedral group. If $H / G_{0}$ contains a dihedral group, $H$ is a direct product of its 2-Sylow subgroup and the Sylow 2-complement. If, moreover, $\phi$ induces a proper L-homomorphism of $\bar{G}_{0}=G_{0} / E_{0}, \bar{G}_{0}$ contains a normal subgroup $\bar{G}_{2}$ such that

(6) $\bar{G}_{0} / \bar{G}_{2}$ is cyclic,

(7) the order of $\bar{G}_{0} / \bar{G}_{2}$ is relatively prime to that of $\bar{G}_{2}$, and

(8) $\phi$ induces an $L$-isomorphism from $\bar{G}_{2}$ into $L$.

As special cases of this theorem we obtain the following theorem.

THEOREM 5. If none of the Sylow complements of a group $G$ is self-conjugate, any $L$-homomorphism from $G$ onto a lattice $L$ is either one of the natural homomorphisms from $L(G)$ onto its direct components, or the L-homomorphism from $G$ onto $G / Z$, where $Z$ is the center of a 2-Sylow subgroup, which is a g.q. group, or combinations of these L-homomorphisms. Hence $L$ is isomorphic to the subgroup lattice of some group.

Since a group $L$-isomorphic to a perfect group is also perfect (cf. [5, Theorem 12]) we obtain the following theorem. 
THEOREM 6. Let $G$ be a perfect group. If $G$ is L-homomorphic to the subgroup lattice $L(H)$ of a group $H$, then $H$ is perfect.

\section{GROUPS $L$-HOMOMORPHIC TO A NILPOTENT GROUP}

In the following two sections we shall consider a homomorphism from the subgroup lattice $L(G)$ of a group $G$ onto $L\left(G^{\prime}\right)$ of another group $G^{\prime}$. We shall call this homomorphism the $L$-homomorphism from $G$ onto $G^{\prime}$. In this section we assume in particular $G^{\prime}$ to be nilpotent, then we can obtain more precise results than those of the preceding section.

Let $G$ be a group and $\phi$ be an $L$-homomorphism from $G$ onto a lattice $L$. Then by Theorem $4, G$ has a normal subgroup $N$ and a subgroup $H$ with properties (1)-(4) of Theorem 4, and if we denote by $E$ or $G_{0}$ the $l$-kernel or the $u$-kernel of $\phi$ respectively, these groups are self-conjugate in $G$. Put $E_{0}$ $=E \cap G_{0}$. These notations will be fixed throughout this section.

LemMA 3. $L(H)$ is directly decomposable if and only if $L$ is directly decomposable.

Proof. If $L(H)$ is directly decomposable, $L$ is clearly decomposable. Assume conversely that $L$ is directly decomposable: $L=L_{1} \times L_{2}$. Then there is a natural homomorphism $\psi_{i}$ from $L$ onto $L_{i}(i=1,2) . \psi_{i} \phi$ is clearly an $L$ homomorphism from $G$ onto $L_{i}$. We shall denote the $l$-kernel of $\psi_{i} \phi$ by $E_{i}$. By Theorem 2, $E_{i}$ is self-conjugate in $G$. We have clearly $E_{1} \cap E_{2}=E$ and $E_{1} \cup E_{2}=G$. When we regard $\psi_{1} \phi$ as an $L$-homomorphism from $G / E$ onto $L_{1}$, the $u$-kernel of $\psi_{1} \phi$ is contained in $E_{2} / E$, and therefore the order of $E_{1} / E$ is relatively prime to that of $E_{2} / E$ by Theorem 4 . Hence $L(G / E)$ is directly decomposable. Since $G / E \cong H / E_{0}$ and since $E_{0} \subseteq \Phi(H)$ by proposition (e) of $\S 2, L(H)$ is also directly decomposable (cf. [5, Lemma 5]). q.e.d.

In the following we shall assume that $L$ is the subgroup lattice of a nilpotent group $G^{\prime}$ and determine the structure of the group $H$. In virtue of Lemma 3, we can assume $G^{\prime}$ to be a $p$-group.

TheOREM 7. Let $G$ be a group, and $\phi$ be an L-homomorphism from $G$ onto a p-group $G^{\prime}$. If $G^{\prime}$ is neither cyclic nor a $P$-group, $H$ is also a p-group and coincides with $G_{0} . G$ is therefore a direct product of $N$ and $G_{0}$. If $G^{\prime}$ is a $P$-group, $H$ is either a p-group or an upper semi-modular group of order $p^{m} q^{n}\left({ }^{10}\right)$, where $q$ is a prime number and $p>q$, and $G_{0}$ is its maximal self-conjugate $M$-group.

Proof. We shall assume that $G_{\bullet}^{\prime}$ is not cyclic. Since $L\left(G^{\prime}\right)$ has no irreducible interval, $H / G_{0}$ is cyclic by Theorem 4 and Lemma 3 . If $\phi$ induces a proper $L$-homomorphism from $G_{0} / E_{0}, G_{0}$ has a normal subgroup $G_{2}$ and $\phi$

(10) Such a group $G$ has been completely determined by Sato [4]. According to him, a group of order $p^{m} q^{n}(p>q)$ is an upper semimodular group if and only if its $p$-Sylow subgroup $P$ is a $P$-group, a $q$-Sylow subgroup $Q$ is cyclic, $Q=\{b\}$, and for any element $a$ of $P, b a b^{-1}=a^{x}$, $x^{q^{t}} \equiv 1(\bmod p)$. 
induces an $L$-isomorphism from $G_{2} / E_{0}$ into $G^{\prime}$. Hence by Theorem 3 of Suzuki [5], $G_{2} / E_{0}$ is a $p$-group or a $P$-group. If $G_{2} / E_{0}$ were a nonabelian $P$-group, $\phi$ would induce an $L$-isomorphism from a group $V / E_{0}$, where $V$ is a subgroup of $G_{0}$, covering $G_{2}$. Since the order of $V / E_{0}$ would be divisible by three distinct primes, this is a contradiction. Hence by proposition $\left(b^{\prime}\right)$ and (d) of $\$ 2$, we see that $H$ is a $p$-group or a group of order $p^{m} q^{n}(p>q)$. If $H$ is a $p$-group, by Lemma 2 we have $H=G_{0}$. We have now only to prove that if the order of $H$ is $p^{m} \cdot q^{n}, H$ is an upper semi-modular group, and $G$ is a $P$-group.

$G_{0} / E_{0}$ is a group of order $p^{\alpha} q^{\beta}$ and its $p$-Sylow subgroup $\bar{S}$ is self-conjugate by Theorem 3 of Suzuki [5] and our Theorem 4. $\phi$ induces an $L$-isomorphism from $\bar{S}$ in to $G^{\prime}$. Take a subgroup $\bar{T}$ of $G_{0} / E_{0}$ covering $\bar{S}^{\prime}$, then $\phi$ induces also an $L$-isomorphism in $\bar{T}$. Hence $T$ is a $P$-group. Next take a $q$-Sylow subgroup $\bar{Q}$ of $G_{0} / E_{0}$ and a subgroup $\bar{V}$ covering $\bar{Q}$; then $\bar{Q}$ is cyclic. Since $G^{\prime}$ is a p-group, $\phi(\bar{V}) \cap \phi(\bar{S})$ is of prime order. Hence $\bar{V} \cap \bar{S}$ is a normal subgroup of $G_{0} / E_{0}$ of order $p$. By direct examination we see that $\phi(\bar{V})$ is a $P$-group. This implies that $G^{\prime}=\phi(\bar{T})$ and $G_{0} / E_{0}=\bar{T}$. Hence we see that $G_{0} / E_{0}$ and $G^{\prime}$ are both $P$-groups.

Since Sylow $p$-complements of $H$ are not self-conjugate, the orders of $H / G_{0}$ and $E_{0}$ are both powers of $q$ by proposition (h) of $\S 2$. The $p$-Sylow subgroup $S$ of $H$ is clearly self-conjugate in $H$ and $\phi$ induces an $L$-isomorphism from $S$ into $G^{\prime}$. Take any subgroup $V$ of order $p$ and any $q$-Sylow subgroup $Q$ of $H$. Then $\phi(V \cup Q)$ is a $P$-group of order $p^{2}$. Hence $(V \cup Q) \cap S$ is of prime order and hence coincides with $V ;(V \cup Q) \cap S=V$. This implies that $V$ is a normal subgroup of $H$. Put $Q=\{b\}$; then for any element $a$ of $S$ we have

$$
b \cdot a \cdot b^{-1}=a^{x}, \quad x \not \equiv 1, \quad x^{q t} \equiv 1(\bmod p) .
$$

Hence $H$ is an upper semi-modular group and $G_{0}$ is its maximal self-conjugate $M$-group. q.e.d.

In order to prove the converse of this theorem we shall first prove the following lemma.

LemMA 4. Let $Z$ be a cyclic subgroup of prime power order contained in the center of a group G. If Sylow subgroups containing $Z$ are cyclic or g.q. groups, the natural homomorphism from $G$ onto $G / Z$ induces an L-homomorphism.

Proof. We can assume that $Z$ is of prime order. We have only to prove $(U \cap V) \cup Z=(U \cup Z) \cap(V \cup Z)$ for any two subgroups $U$ and $V$ of $G$. If $U \supseteq Z$ and $V \supseteq Z$, we have clearly this equality. If $U \nsupseteq Z$, the order of $U$ is prime to $p$. Hence we have $L(U \cup Z)=L(Z) \times L(U)$ (cf. [3]). If moreover $V \supseteq Z$, we have $(U \cup Z) \cap V=Z \cup(((U \cup Z) \cap V) \cap U)=Z \cup(U \cap V)$. If $V \nsupseteq Z$, $(U \cup Z) \cap(V \cup Z)=Z \cup W$ for some subgroup $W$. We have then $U \cap V \supseteq W$. Hence we have $(U \cup Z) \cap(V \cup Z) \subseteq(U \cap V) \cup Z$. On the other hand, we have 
clearly $(U \cap V) \cup Z \subseteq(U \cup Z) \cap(V \cup Z)$. Hence $(U \cap V) \cup Z=(U \cup Z)$ $\cap(V \cup Z)$. q.e.d.

If a group $G$ is a direct product of two groups $G_{0}$ and $N$ (having relatively prime orders), and if $G_{0}$ is a p-group, $G$ is clearly $L$-homomorphic to $G_{0}$. If $H$ is an upper semi-modular group and $G_{0}$ is its maximal self-conjugate $M$-group, $G$ is $L$-homomorphic to a $P$-group as follows. First the mapping $U \rightarrow U \cup E_{0}$ from $L(G)$ onto $L\left(G / E_{0}\right)$ is surely an $L$-homomorphism by Lemma 4 . Hence we may assume that $E_{0}=e$. As $H$ is an upper semi-modular group, the mapping $U \rightarrow U \cap G_{0}$ from $L(H)$ onto $L\left(G_{0}\right)$ is an $L$-homomorphism. We shall prove that the mapping $U \rightarrow U \cap G_{0}(U \subseteq G)$ is also an $L$-homomorphism from $G$ onto $G_{0}$. First we shall show that $\left(U \cap G_{0}\right) \cup N=(U \cup N) \cap\left(G_{0} \cup N\right)$ for any subgroup $U$ of $G$. Suppose that the order of $U$ is $p^{\alpha} q^{\beta} g,(p q, g)=1$. If $\beta=0$, $U$ is contained in $S \cup N$, where $S$ is a $p$-Sylow subgroup of $G$. Since $L(S \cup N)$ $=L(S) \times L(N)$, we have easily $\left(U \cap G_{0}\right) \cup N=(U \cup N) \cap\left(G_{0} \cup N\right)$. If $\beta \neq 0$, the index $\left[\left(U \cap G_{0}\right) \cup N: N\right]$ is equal to $p^{\alpha} q$, and $\left[(U \cup N) \cap\left(G_{0} \cup N\right): N\right]$ is also equal to $p^{\alpha} q$. On the other hand, we have $\left(U \cap G_{0}\right) \cup N \subseteq(U \cup N) \cap\left(G_{0} \cup N\right)$. Hence we have $\left(U \cap G_{0}\right) \cup N=(U \cup N) \cap\left(G_{0} \cup N\right)$.

Now we shall show that $(U \cup V) \cap G_{0}=\left(U \cap G_{0}\right) \cup\left(V \cap G_{0}\right)$. In fact, we have

$$
N \cup\left((U \cup V) \cap G_{0}\right)=(U \cup V \cup N) \cap\left(G_{0} \cup N\right) .
$$

On the other hand, as $G / N$ is an upper semi-modular group,

$$
\begin{aligned}
((U \cup N) \cup(V \cup N)) & \cap\left(G_{0} \cup N\right) \\
& =\left((U \cup N) \cap\left(G_{0} \cup N\right)\right) \cup\left((V \cup N) \cap\left(G_{0} \cup N\right)\right) \\
& =\left(\left(U \cap G_{0}\right) \cup N\right) \cup\left(\left(V \cap G_{0}\right) \cup N\right) \\
& =\left(\left(U \cap G_{0}\right) \cup\left(V \cap G_{0}\right)\right) \cup N .
\end{aligned}
$$

Since $G_{0} \cap N=e$, we have

$$
\begin{aligned}
(U \cup V) \cap G_{0} & \cong N \cup\left((U \cup V) \cap G_{0}\right) / N \\
& \cong\left(\left(U \cap G_{0}\right) \cup\left(V \cap G_{0}\right)\right) \cup N / N \cong\left(U \cap G_{0}\right) \cup\left(V \cap G_{0}\right) .
\end{aligned}
$$

Hence we have $(U \cup V) \cap G_{0}=\left(U \cap G_{0}\right) \cup\left(V \cap G_{0}\right)$. The mapping $U \rightarrow U \cap G_{0}$ is thus an $L$-homomorphism from $G$ onto a $P$-group $G_{0}$.

From Lemmas 1 and 3, Theorem 7, and the remark given above we obtain:

THEOREM 8. Let $G$ be a group. There exists an L-homomorphism $\phi$ from $G$ onto a nilpotent group $G^{\prime}=\prod_{i=1}^{i} S_{i}$, where $S_{i}$ is a $p_{i}$-Sylow subgroup of $G^{\prime}$, if and only if $G$ has a normal subgroup $N$ and a subgroup $H$ with the following properties:

(1) $N H=G$ and $N \cap H=e$.

(2) the order of $N$ is relatively prime to that of $H$, 
(3) $H$ is a direct product of groups $H_{i}(i=1,2, \cdots, t)$ having mutually prime orders: $H=\prod_{i=1}^{t} H_{i}$,

(4) $\phi\left(H_{i}\right)=S_{i}(i=1,2, \cdots, t)$,

(5) if $S_{j}$ is cyclic, $H_{j}$ is a cyclic group of prime power order or a g. q. group, and $H_{j}$ contains a normal subgroup $K_{j}$ of $G$ such that $\phi\left(K_{j}\right)=S_{j}$,

(6) if $S_{k}$ is a P-group of order $p_{k}^{n+1}(n \geqq 1), H_{k}$ is either isomorphic to $S_{k}$, or a quaternion group $\left(n=1, p_{k}=2\right)$, or an upper semi-modular group of order $p_{k}^{n} q^{m}$ ( $q$ is a prime and $p_{k}>q$ ), and its maximal self-conjugate $M$-group is a normal subgroup of $G$,

(7) if $S_{l}$ is neither cyclic nor a $P$-group, $H_{l}$ is also a p proup and selfconjugate in $G$. In this case if $H_{l}$ is not L-isomorphic to $S_{l}, H_{l}$ is a g.q. group and $S_{l}$ is isomorphic to the factor group $H_{l} / Z_{l}$ of $H_{l}$ modulo its center $Z_{l}$.

We shall omit the proof of this theorem, since it runs along similar lines as the proof of Theorem 1 .

\section{The $L$-HOMOMORPHIC IMAge OF A SOLVABLE GROUP}

In this section we shall prove the following theorem.

THEOREM 9. Let $G$ be a solvable group, and $\phi$ be an L-homomorphism from $G$ onto another group $G^{\prime}$. Then $G^{\prime}$ is also solvable.

Denote by $E$ or $G_{0}$ the $l$-kernel or the $u$-kernel of $\phi$ respectively. Then by Theorems 2 and $3, E$ and $G_{0}$ are self-conjugate. Put $E_{0}=E \cap G_{0} . \phi$ induces an $L$-homomorphism $\phi$ from $G_{0} / E_{0}$ onto $G^{\prime}$. If $\phi$ is an $L$-isomorphism, our theorem follows from a theorem on the $L$-isomorphism which asserts that groups $L$-isomorphic to a solvable group are also solvable (cf. [5, Theorem 12]). If $\phi$ is a proper $L$-homomorphism, $G_{0} / E_{0}$ contains a normal subgroup $G_{2} / E_{0}$ such that $G_{0} / G_{2}$ is cyclic and $\phi$ induces an $L$-isomorphism from $G_{2} / E_{0}$ into $G^{\prime}$. Hence in order to prove our Theorem 9 , it is sufficient to prove the following theorem.

Theorem 10. Assume $L$ to be a lattice of subgroups of a group $G^{\prime}$. Then under the same notations as in Theorem $4, \phi\left(G_{2}\right)$ is self-conjugate in $G^{\prime}$.

Proof. In changing the notations, we shall assume that the $u$-kernel of $\phi$ coincides with $G$ and that the $l$-kernel of $\phi$ coincides with $e$. Take a $p$-Sylow subgroup $S$ of $G$ in which $\phi$ induces a proper $L$-homomorphism. By Lemma 3 and proposition $(\mathrm{g})$ of $\S 2, S$ must be cyclic, and by proposition (h) of $\S 2, G$ has a Sylow $p$-complement $N$. We shall first prove that $\phi(S)$ is also a Sylow subgroup of $G$.

Since $\phi(S)$ is a cyclic group of prime power order, it is contained in some Sylow subgroup $S^{\prime}$ of $G^{\prime}$. Take the greatest subgroup $U$ of $G$ such that $\phi(U)=S^{\prime}$. Then $U$ clearly contains $S$. If $S^{\prime}$ were a $P$-group, $\phi(S)$ would be of prime order. On the other hand, taking the maximal subgroup $M$ of $S$, 
we have $\phi(M) \neq \phi(S)$, as the $u$-kernel of $\phi$ coincides with $G$. Hence we would have $\phi(M)=e$, that is, $M$ would be contained in the $l$-kernel of $\phi$ and by our assumption $M=e$. Hence $S$ is mapped $L$-isomorphically onto $\phi(S)$, contrary to our assumption. Hence $S^{\prime}$ is not a $P$-group and $U$ is also of prime power order by Theorem 8 . Hence $U$ must coincide with $S$, that is, $S^{\prime}=\phi(S)$.

Next we shall prove that $S^{\prime}=\phi(S)$ is contained in the center of its normalizer. Take a subgroup $V^{\prime}$ of $G^{\prime}$ such that $S^{\prime}$ is self-conjugate in $V^{\prime}$, and $V^{\prime} / S^{\prime}$ is of prime power order, say of order $q^{n}$ ( $q$ is a prime number). Take a subgroup $V$ of $G$ such that $\phi(V)=V^{\prime}$; then $\phi(V \cap N)$ is a $q$-Sylow subgroup $Q^{\prime}$ of $V^{\prime}$. If $V \cap N$ is cyclic and not $L$-isomorphic to $Q^{\prime}, S$ is self-conjugate in $V$ by proposition (h) of $\S 2$, and hence $V$ and also $V^{\prime}$ are directly decomposable.

We can then assume $V \cap N$ to be $L$-isomorphic to $\left.Q^{\prime}{ }^{11}\right)$. Since the $l$-kernel of $\phi$ coincides with $e$, a subgroup $T$ of $V$, covering $N \cap V$, is $L$-isomorphic to $\phi(T)=T^{\prime}$, and $\phi$ induces an $L$-isomorphism from $T$ onto $T^{\prime}$. By our assumption, $T^{\prime} \cap S^{\prime}$ is self-conjugate in $T^{\prime}$. If $T \cap S$ were not self-conjugate in $T, T$ would be a $P$-group (cf. [5, Theorems 13 and 14]) which would imply that $Q^{\prime}$ has prime order. Hence $V \cap N$ would also be of prime order. Since $\phi(S)$ is self-conjugate in $V^{\prime}, V^{\prime}$ is a $P$-group, which leads us to the same contradiction as above. Hence $T \cap S$ is self-conjugate in $T$ and so $T$ is a direct product of $N \cap V$ and $T \cap S$. This implies that $T \cap S$ is self-conjugate in $V$. If $S$ were not self-conjugate in $V$, there would be another $p$-Sylow subgroup $S^{*}$ of $V$. $S^{*}$ would also contain $T \cap S$. Hence we would have $\phi\left(S^{*}\right) \cap S^{\prime} \neq e$. Since $\phi\left(S^{*}\right)$ is a cyclic group of prime power order, this gives a contradiction. Hence we have $V=(N \cap V) \times S$ and $V^{\prime}=Q^{\prime} \times S^{\prime} . S^{\prime}$ is thus contained in the center of its normalizer and $G^{\prime}$ contains a normal subgroup $N^{\prime}$ such that $N^{\prime} S^{\prime}=G$ and $N^{\prime} \cap S^{\prime}=e\left({ }^{12}\right)$.

We shall now prove that $\phi(N)=N^{\prime}$. Take all $p$-Sylow subgroups $S=S_{1}$, $S_{2}, \cdots, S_{t}$ of $G$. Then $\phi$ induces a proper $L$-homomorphism in every $S_{i}$. Hence the $\phi\left(S_{i}\right)$ are Sylow subgroups of $G^{\prime}$ and are contained in centers of their normalizers, as proved above. $G$ then has Sylow complements $N^{\prime}=N_{1}^{\prime}$, $N_{2}^{\prime}, \cdots, N_{r}^{\prime}$. Put $D^{\prime}=\bigcap_{i=1}^{r} N_{i}^{\prime}$. Take a subgroup $D$ of $G$ such that $\phi(D)=D^{\prime}$. Since $D^{\prime} \cap \phi\left(S_{i}\right)=e(i=1,2, \cdots, t)$, we have $D \cap S_{i}=e(i=1,2, \cdots, t)$, which implies that the order of $D$ is prime to $p$, or $D \subseteq N$. Since $\phi(N) \supseteq D^{\prime}$, $\phi(N) \cap \phi(S)=e$ and $\phi(N) \cup \phi(S)=G^{\prime}$, we have $\phi(N)=N^{\prime}$. This proves our theorem.

\section{Neutral Elements of $L(G)$}

An element $l$ of a lattice $L$ is called neutral if every triple $\{l, x, y\}$ of elements of $L$ generates a distributive sublattice of $L$. An element $l$ of $L$ is neutral if and only if the mappings $x \rightarrow x \cup l$ and $x \rightarrow x \cap l$ are homomorphisms, and $x \cup l=y \cup l$ and $x \cap l=y \cap l$ imply $x=y$ for any two elements $x, y$ of $L$

(11) Cf. Theorem 7.

(12) By Burnside's theorem, cf. Zassenhaus [7, p. 131]. 
(Birkhoff [1]). If $L$ is directly decomposable, an element is neutral if and only if all its components are neutral.

In this section we shall determine the neutral elements of a subgroup lattice $L(G)$ of a group $G$. Because of the above remark we may assume $L(G)$ to be irreducible.

Let $K$ be a neutral element of $L(G)$. Then the mapping $\phi: U \rightarrow U \cup K$ is an $L$-homomorphism from $G$ onto an interval $G / K$. As $K$ is the $l$-kernel of $\phi$, it is self-conjugate in $G$ by Theorem 2. Denote the $u$-kernel of $\phi$ by $G_{0}$; then we have $G_{0} \cup K=G$. By proposition (c) of $\S 2$, we have either $G_{0} \supseteq K$ or $L(G)$ is directly decomposable. Hence from our assumptions we have $G_{0} \supseteq K$, so $G_{0}=G$. By Theorem $4, K$ is a cyclic group contained in the center of $G$. On the other hand, the mapping $U \rightarrow U \cap K$ is also an $L$-homomorphism from $G$ onto $K$. Since $K$ is cyclic, the structure of $G$ is determined by Theorem 1 . Let $K=\prod_{i=1}^{t} K_{i}$ be the decomposition of $K$ into a direct product of its Sylow subgroups $K_{i}$. Then $G$ has a normal subgroup $N$ and a subgroup $H$ with the following properties:

(1) $N H=G, N \cap H=e$, and $H \supseteq K$,

(2) the order of $N$ is prime to that of $H$,

(3) $H$ is a direct product $\prod_{i=1}^{t} H_{i}$ of its Sylow subgroups $H_{i}$, and

(4) $H_{i}$ is either cyclic or a g.q. group.

Conversely suppose that a subgroup $K$ of a group $G$ is contained in the center of $G$ and $G$ has a normal subgroup $N$ and a subgroup $H$ with the properties (1)-(4) given above. Then $K$ is a neutral element of $L(G)$.

Proof. By (4), $K$ is cyclic. Let $K_{i}$ be a $p_{i}$-Sylow subgroup of $K$. We shall show that $K_{i}$ is neutral. By Lemma 4, the mapping $U \rightarrow U \cup K_{i}$ is an $L$-homomorphism from $G$ onto $G / K_{i}$. By Lemma 1, the mapping $U \rightarrow U \cap K_{i}$ is also an $L$-homomorphism from $G$ onto $K_{i}$. We have only to prove that $U \cup K_{i}$ $=V \cup K_{i}$ and $U \cap K_{i}=V \cap K_{i}$ imply $U=V$ for any two subgroups $U, V$ of $G$. $G$ has a Sylow $p_{i}$-complement $N_{i}$. We have $U \supseteq K_{i}$, or $U \subseteq K_{i} N_{i}$ for any subgroup $U$ of $G$. Suppose now that $U \cup K_{i}=V \cup K_{i}$ and $U \cap K_{i}=V \cap K_{i}$. If $U \supseteq K_{i}$, we have $U \cap K_{i}=K_{i}$. Hence we have $V \cap K_{i}=K_{i}$, or $V \supseteq K_{i}$. We have, therefore, $U=U \cup K_{i}=V$. If $U \supseteq K_{i}$, we have also $V \supseteq K_{i}$, that is, $N_{i} K_{i}$ contains both $U$ and $V$. On the other hand, $N_{i} K_{i}$ is a direct product of $N_{i}$ and $K_{i}$, and we have $L\left(N_{i}, K_{i}\right)=L\left(N_{i}\right) \times L\left(K_{i}\right)$. Hence we have clearly

$$
\begin{aligned}
U & =\left(U \cap K_{i}\right) \cup\left(U \cap N_{i}\right)=\left(U \cap K_{i}\right) \cup\left(\left(U \cup K_{i}\right) \cap N_{i}\right) \\
& =\left(V \cap K_{i}\right) \cup\left(\left(V \cup K_{i}\right) \cap N_{i}\right)=V .
\end{aligned}
$$

Since the join of neutral elements is also neutral, $K=\bigcup_{i=1}^{t} K_{i}$ is neutral. Thus we obtain the following theorem, which gives an answer to a problem of Birkhoff $\left({ }^{13}\right)$.

Theorem 11. Assume that the subgroup lattice $L(G)$ of a group $G$ is ir-

${ }^{(13)}$ Problem 35, described in the revised edition of his book Lattice theory. 
reducible. A subgroup $K$ of $G$ is a neutral element of $L(G)$ if and only if $K$ is contained in the center of $G$, and $G$ has a normal subgroup $N$ and subgroup $H$ with the properties (1)-(4) given above.

Added in proof. After writing this paper, the author learned that G. Zappa has obtained some theorems concerning $L$-homomorphisms of finite groups, in particular Theorem 1 of this paper: Cf. G. Zappa, Determinazione dei gruppi finiti in omomorfismo strutturale con un gruppo ciclico, Rendiconti del seminario Matematico, Univ. di Padova (1949) pp. 140-162, and Sulla condizione perche un omomorfismo ordinario sia anche un omomorfismo strutturale, Giornale di Matematiche vol. 78 (1949) pp. 182-192.

For the detailed proof of a theorem of N. Ito, cited in footnote 7 of this paper, see his forthcoming paper: Note on $(L M)$-groups of finite orders, Kôdai Mathematical Seminar Reports.

\section{BiBliograPHY}

1. G. Birkhoff, Neutral elements in general lattices, Bull. Amer. Math. Soc. vol. 46 (1941) pp. 702-705. 1940.

2. - Lattice theory, Amer. Math. Soc. Colloquium Publications, vol: $\dot{j}_{25}$, New York,

3. K. Iwasawa, Über die Gruppen und die Verbände ihrer Untergrupen, '̛. Fac. Sci. Imp. Univ. Tokyo. Sect. I, IV-3 (1941) pp. 171-199.

4. S. Sato, On groups and the lattices of subgroups, Osaka Mathematical Journal vol. 1 (1949) pp. 135-149.

5. M. Suzuki, On the lattice of subgroups of finile groups, Trans. Amer. Math. Soc. vol. 70 (1951) pp. 345-371.

6. P. M. Whitman, Groups with a cyclic group as lattice-homomorph, Ann. of Math. vol. 49 (1948) pp. 347-351.

7. H. Zassenhaus, Lehrbuch der Gruppentheorie I, 1937.

ToKyo University, TOKYo, JAPAN. 